1 Watford, UK

2 Stort Valley and Villages Primary Care Network

3 Imperial College London, London, UK

Twitter @DrSimonHodes @Azeem_Majeed @SianStanley1 Cite this as: BMJ 2022;376:0338 http://dx.doi.org/10.1136/bmj.0338

Published: 07 February 2022

\section{A national vaccination service for the NHS in England: a proposal to be considered with caution}

\author{
A separate national vaccination service risks further fragmenting NHS primary care in England \\ Simon Hodes, ${ }^{1}$ Sian Stanley, ${ }^{2}$ Azeem Majeed ${ }^{3}$
}

The health secretary, Sajid Javid, announced on 26 January that a "national vaccination service" is required to provide mass covid-19 vaccination to the population of England. ${ }^{1}$ Speaking at a House of Commons Health and Social Care Committee meeting, Javid suggested the proposed service could cover other vaccines as well as vaccines for covid-19. The rationale is that NHS general practice is under great strain, and by removing some services that can be provided elsewhere, it will free up time for primary care teams to concentrate on their core work.

Traditionally, mass vaccine programmes in England have relied largely on general practices, increasingly supported by community pharmacies in recent years. This was demonstrated to great effect during the first wave of covid-19 vaccinations where the majority of vaccines were delivered by primary care teams. GP teams have secure electronic patient record systems, and are experienced in cold storage chains, and have medical support on site, including resuscitation equipment. Patients often know and trust their family doctors, and generally respond better to recalls for vaccination when these come from their own general practices. A move towards mass vaccine centres and away from primary care delivery may explain some of the recent slow-down in England's covid-19 vaccine programme. $^{2}$

The public need to be fully informed about what a national vaccination service will mean for them individually as well as the NHS. The majority of all NHS contacts occur in general practice, with around one million contacts per day. ${ }^{3}$ This means that vaccines can be offered opportunistically when patients are attending for other reasons as well as in dedicated vaccine clinics. It also allows primary care teams to have discussions about vaccination during these consultations in patients who have concerns or questions about vaccines, or who are vaccine hesitant.

When attending for vaccination, patients also have the opportunity to discuss other issues in their health with their primary care team and to benefit from opportunistic health promotion. All this helps to ensure that vaccination is viewed holistically and not just as a transactional activity. This is particularly important for children where non-attendance for vaccination can sometimes be a safeguarding issue which requires a sensitive approach from primary care teams, as well as effective inter-agency working.

When the prime minister, Boris Johnson, announced that he wanted all adults in England to be offered a covid-19 vaccine before the end of 2021, he looked to GPs to help. As a result, GPs were asked to drop all non-essential work and focus on vaccination for the remainder of the year. This caused much debate in the national and medical press about what the priorities should be for the NHS and for primary care. Suspending "non-essential work" will have adverse effects on people's experience of the NHS and risks worsening health outcomes, particularly for poorer groups. ${ }^{4}$ It is clearly also a policy that cannot be sustained for long or repeated frequently (for example, for another covid-19 vaccine booster programme later this year).

The current plan to consider a separate national vaccination service for covid-19 and possibly other vaccinations seems to be an effort to ensure that GPs are not asked to stop routine medical care again. Although investment in the NHS is welcome-and removing some workload from general practice might have merits-there are some caveats that must be considered before a new national vaccination service is established.

Firstly, any new vaccination service must be more cost effective than existing models of delivery of vaccines, such as through general practices and great pressure, NHS funding must be used cost-effectively and services delivered efficiently. A new national vaccination service would require substantial funding to establish and run. For example, it is difficult to see how a national vaccine service could run effectively without full access to patients' electronic medical records. It would also require premises from which to operate, and staff to manage and deliver the programme. We need the government to show how this investment in a new service would compare in terms of cost effectiveness with a similar investment in primary care teams.

Secondly, a national vaccination service must achieve a high uptake of vaccination. We currently have very and in 2021-22, primary care teams also achieved a record uptake of flu vaccines, for an extended group of patients compared to previous years. Vaccinations must also be delivered quickly and at scale when in a pandemic, and there must be a safe and robust system to target high risk groups; such as those with frailty, long term conditions, the housebound, people living care homes, and patients from marginalised groups. 5

Thirdly, creating a separate vaccination service risks further fragmentation of primary care. As we have already seen with the covid-19 NHS 119 service, many patients will still contact their GPs about vaccination queries, even if this is no longer part of the NHS GP pharmacists. At a time when NHS budgets are under good uptake of most childhood vaccines in England 
contract. This risks creating extra work for primary care teams that is not part of their core contract and for which they will not be paid; and will also be very frustrating for patients who will have to deal with more than one healthcare provider to have any issues they have about their vaccinations and how these vaccinations are recorded are dealt with. Finally, a newly established national vaccine service may recruit staff from primary care teams, both clinical and non-clinical, thereby further worsening the current shortages of staff in NHS primary care. ${ }^{6}$

The government must therefore carefully examine the merits of a separate national vaccination service; and any problems it may cause for existing services; including how it might affect vaccine uptake. Investing in and strengthening existing NHS primary care infrastructure in general practices and pharmacies may be a more cost effective option. Because of the importance of vaccination in allowing England to move to "living with covid-19," vaccinations programmes must be implemented well and achieve a high take-up, particularly in the groups most at risk of serious illness,

compilations, and death from infectious diseases such as covid-19. We cannot risk undermining the current vaccination systems that already work efficiently and cost-effectively in England's NHS. Any proposals for a new national vaccination service must therefore be assessed with the same rigour we would with any new medical treatment with serious consideration of the risks as well as the benefits.

Competing interests: We have read and understood the BMJ policy on declaration of interests. We are all GPS and have supported the NHS covid-19 vaccination programme. We have no other competing interests.

Acknowledgments: AM is supported by the NIHR Applied Research Collaboration NW London. The views expressed are those of the authors and not necessarily those of the NHS, the NIHR or the Department of Health and Social Care.

1 Health secretary proposes 'national vaccination service' to relieve GPs. https://www.pulsetoday.co.uk/news/breaking-news/health-secretary-proposes-national-vaccination-service-to-offloadgps/

2 Where are we with covid-19 vaccination in the United Kingdom? https://blogs.bmj.com/bmj/2021/07/09/where-are-we-with-covid-19-vaccination-in-the-unitedkingdom/

3 Appointments in General Practice. https://digital.nhs.uk/data-and-information/publications/statistical/appointments-in-general-practice-weekly-mi/current

4 Majeed A, Maile EJ, Bindman AB. The primary care response to COVID-19 in England's National Health Service. J R Soc Med 2020;113:208-10. doi: 10.1177/0141076820931452 pmid: 32521196

5 Covid-19 vaccines: patients left confused over rollout of third primary doses. https://blogs.bmj.com/bmi/2021/10/15/covid-19-vaccines-patients-left-confused-over-rollout-ofthird-primary-doses/

6 Oliver D. David Oliver: Act on workforce gaps, or the NHS will never recover. BMJ2022;376:n3139. doi: 10.1136/bmj.n3139 pmid: 34987050 